HYPERTENSION

\section{Global blood pressure trends}

High blood pressure, a leading global risk factor for chronic kidney disease, is an important health issue worldwide. A new study shows that mean blood pressure has increased in low-income countries in the past 40 years. "This type of comparative performance data will allow us to investigate the determinants of high versus low blood pressure and guide the choice and evaluation of policies to lower blood pressure," says Majid Ezzati, who designed the study.

To understand how blood pressure levels have changed in different countries over time, the researchers assembled a global network of researchers and practitioners who analysed blood pressure levels in 19.1 million adults aged $>18$ years from 1,479 population-based studies in 200 countries from 1975 to 2015. "We pooled the data using a bespoke Bayesian statistical model that was specifically designed to analyse cardiometabolic factors such as blood pressure," explains Ezzati.

High blood pressure, which used to affect mainly high-income countries, is now most prevalent in low-income countries in South Asia and sub-Saharan Africa, and has remained high in central and Eastern Europe. "These results mean that we should abandon the notion of high blood pressure as a condition of affluence and treat it as one of poverty, and as highest in the global low-income population," says Ezzati.

Ezzati and colleagues also found that the number of people with raised blood pressure in the world had increased by $90 \%$ over the four decades; the increase was largest in countries with middle and low incomes and stemmed largely from growth and ageing of the population.

In the future, the researchers will continue to monitor global trends in key cardiometabolic risk factors. "In addition, we hope that the results of this study, which are freely and openly available online for use by everyone (www.ncdrisc.org), will provide a resource to understand the drivers of variations across countries, and hence provide best-practice lessons."

Andrea Aguilar

ORIGINAL ARTICLE NCD risk factor collaboration (NCD-RisC). Worldwide trends in blood pressure from 1975 to 2015: a pooled analysis of 1479 population-based measurement studies with $19 \cdot 1$ million participants. Lancet http://dx.doi.org/10.1016/S0140-6736(16)31919-5 (2016) 